\title{
CRISPR-Cas13d for Gene Knockdown and Engineering of CHO Cells
}

Chih-Che Shen ${ }^{1}$, Mei-Wei Lin ${ }^{1,2}$, Bao Khanh Thi Nguyen ${ }^{1}$, Chin-Wei Chang ${ }^{1}$, Jie-Ru Shih ${ }^{3}$, Mai Thanh Thi Nguyen ${ }^{4}$, Yi-Hao Chang ${ }^{1}$ and $\mathrm{Yu}-\mathrm{Chen} \mathrm{Hu}^{1,5^{*}}$

${ }^{1}$ Department of Chemical Engineering, National Tsing Hua University, Hsinchu, Taiwan 30003

${ }^{2}$ Biomedical Technology and Device Research Laboratories, Industrial Technology Research Institute, Hsinchu, Taiwan 30003

${ }^{3}$ Mycenax Biotech Inc., Miaoli, Taiwan 35053

${ }^{4}$ Faculty of Chemistry, University of Science, Vietnam National University Ho Chi Minh City, Ho Chi Minh City, Vietnam 72711

${ }^{5}$ Frontier Research Center on Fundamental and Applied Sciences of Matters, National Tsing Hua University, Hsinchu, Taiwan 30003

Running Title: CRISPR-Cas13d for CHO cell engineering

*Corresponding Author

Phone: (886)3-571-8245

FAX: (886)3-571-5408

Email: yuchen@che.nthu.edu.tw 
(A)
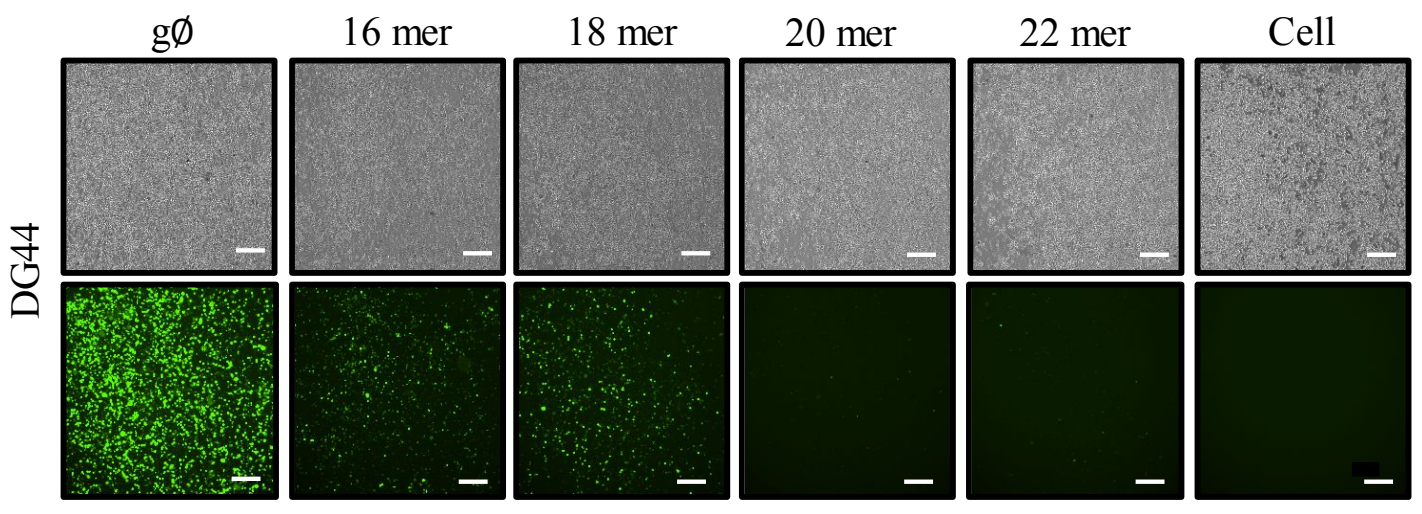

(B)

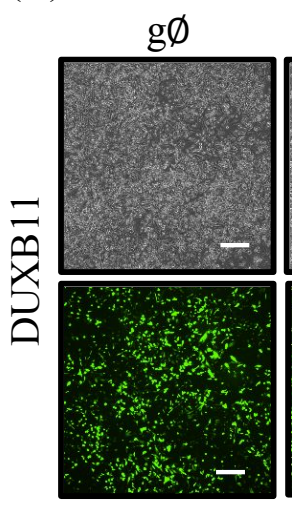

(C)

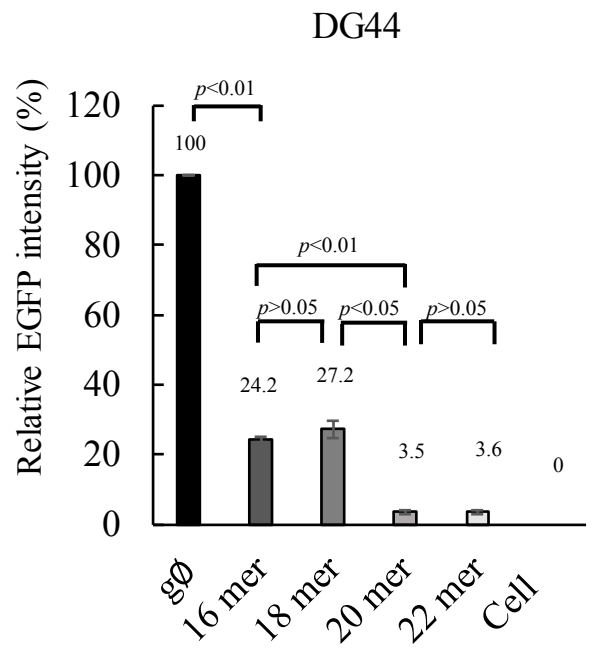

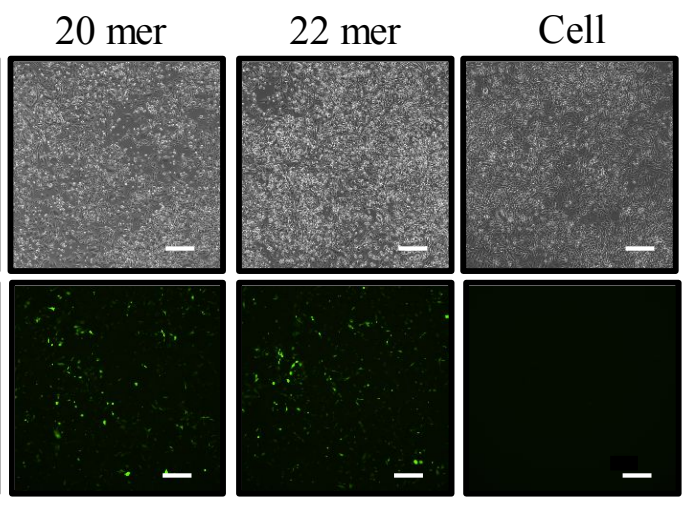

(D)

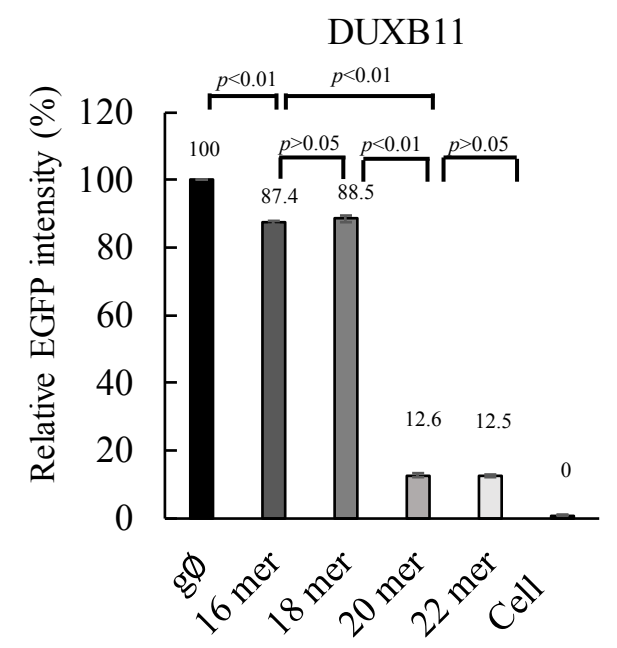

Figure S1. CRISPR-Cas13d suppressed exogenous gene expression in CHO DG44 and DUXB11 cells. (A) Fluorescence micrographs of DG44 cells. (B) Fluorescence micrographs of DUXB11 cells. (C) Relative EGFP intensity in DG44 cells. (D) Relative EGFP intensity in DUXB11 cells. DG44 and DUXB11 cells were transfected with pDHFR-EGFP and pEF1 $\alpha$-Cas13dPZ-gRNA $A_{\text {DHFR }}$ (or pEF1 $\alpha$-Cas13dPZ-gRNA $\varnothing$ ) as described in Fig. 1. Non-transfected cells (Cell group) served as the negative control. The data showed that co-transfection of pEF1 $\alpha$-Cas13dPZ-gRNA ${ }_{\text {DHFR }}$ and pDHFR-EGFP into DG44 and DUXB11 resulted in effective gene suppression. The knockdown efficiency was dependent on the spacer length and 22 mer was the most effective. Bar, $500 \mu \mathrm{m}$. 
(A)

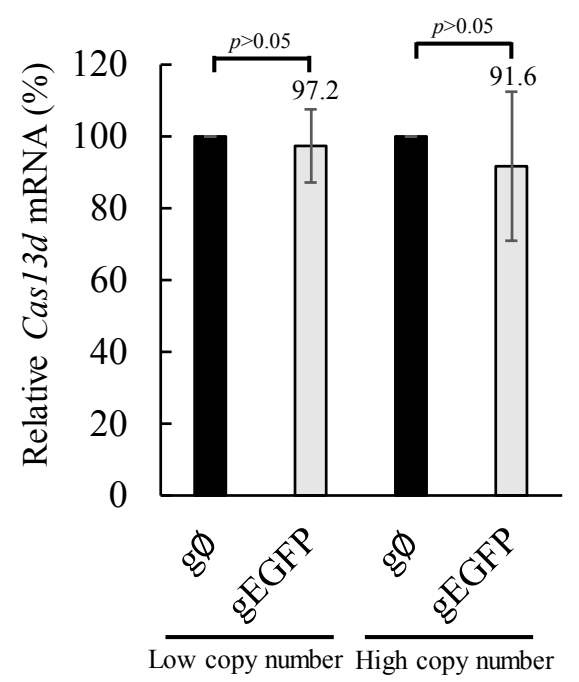

(B)

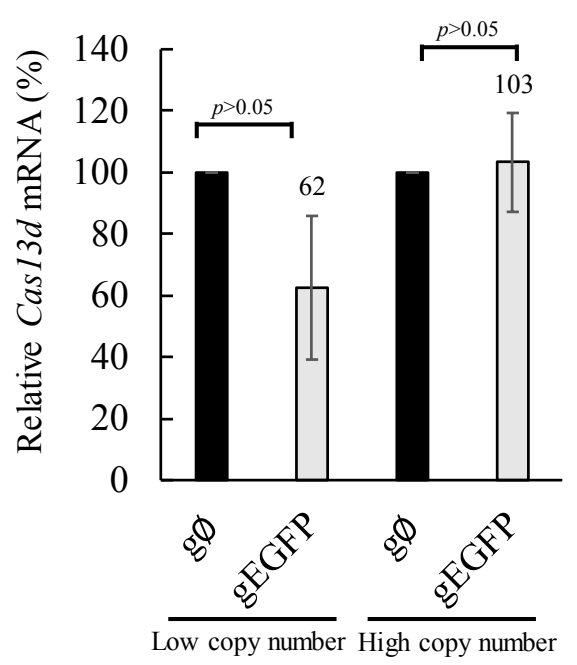

Figure S2. Cas13d expression level in CHO DUXB11 cells harboring low or high copy number of EGFP. (A) Relative Cas13d expression levels after transfection. (B) Relative Cas13d expression levels after transfection and Zeocin selection. The experiments were performed as in Fig. 2 and the data in the gEGFP group were normalized to those of the $\mathrm{g} \varnothing$ group. The data show that the Cas $13 \mathrm{~d}$ expression levels were statistically similar between the gEGFP and gØ groups. 
pCRISPRi-gRNA

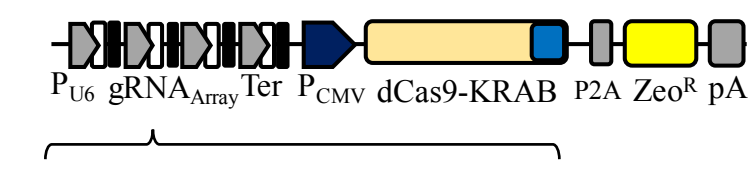

g1g2g3g4 g5g6g7g8 g9g10g11g12

g13g14g15g16 g17g18g19g20 g21g22g23g24

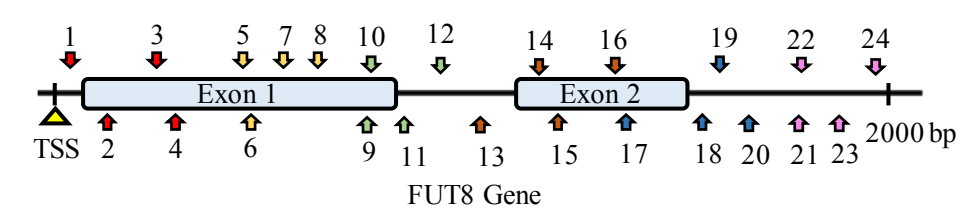

dCas9 gRNA Target site

(D)

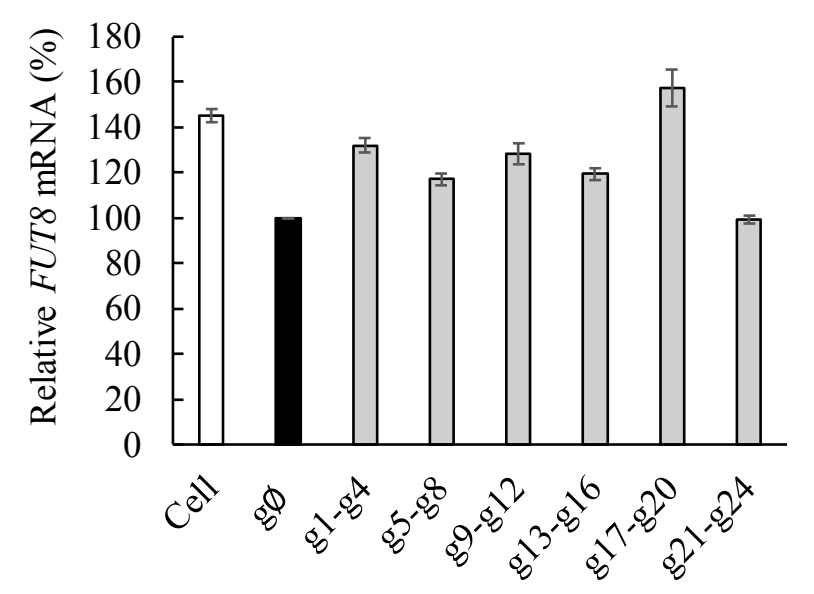

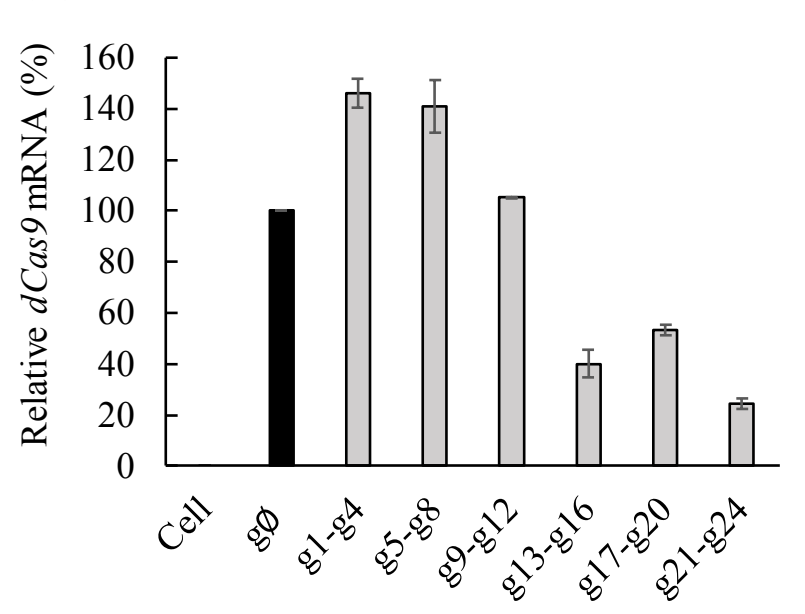

Figure S3. FUT8 gene repression using the dCas9-KRAB-based CRISPRi system was not effective.

(A) Schematic of the pCRISPRi-gRNA expression vectors. pCRISPRi-gRNA expressed dCas9KRAB and the gRNA array comprising 4 gRNA in tandem. A total of 24 gRNA targeting 24 sites of FUT8 gene were designed. (B) Targeting sites on FUT8 gene. (C) FUT8 gene expression. (D) dCas9 expression level. $\mathrm{CHO}_{\mathrm{IgG}}$ cells were transfected with the pCRISPRi-gRNA plamids and the expression levels were analyzed by qRT-PCR. The expression levels were analyzed using Beta-2microglobulin (B2M) gene as the internal control and normalized to the gØ group (expressing the nontargeting gRNA). Non-transfected cells were used as the Cell control. The data were representative of averages of 3 independent culture experiments and showed that the dCas9-KRAB system was unable to effectively suppress FUT8 expression (C). 
(A)

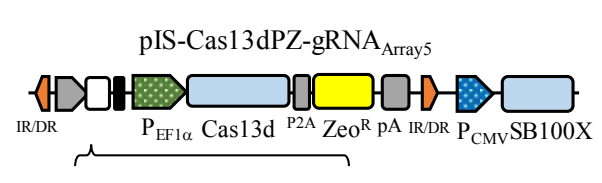

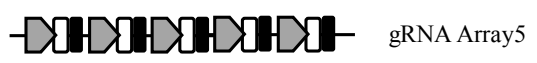

$\mathrm{BAK}_{\mathrm{gRNA}} \mathrm{BAX}_{\mathrm{gRNA} 1,2} \mathrm{PDK}_{\mathrm{gRNA}}, 2$
(B)

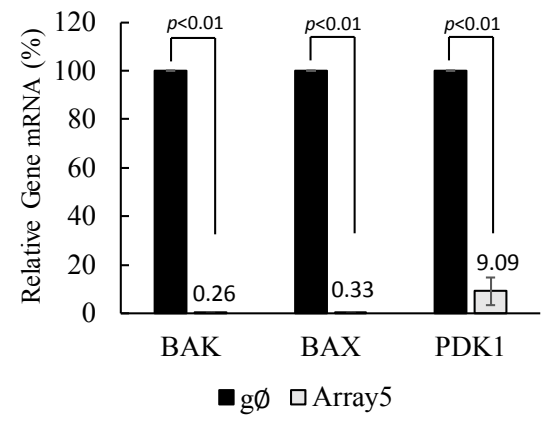

(C)

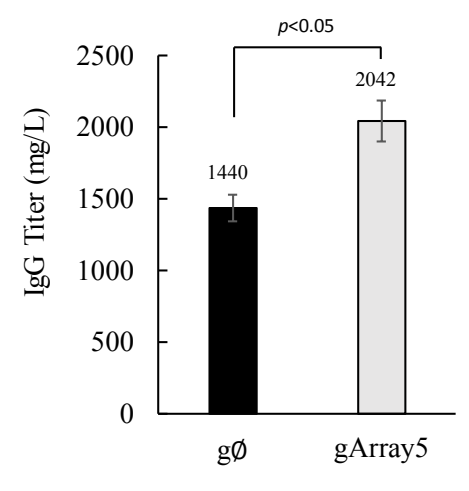

(D)

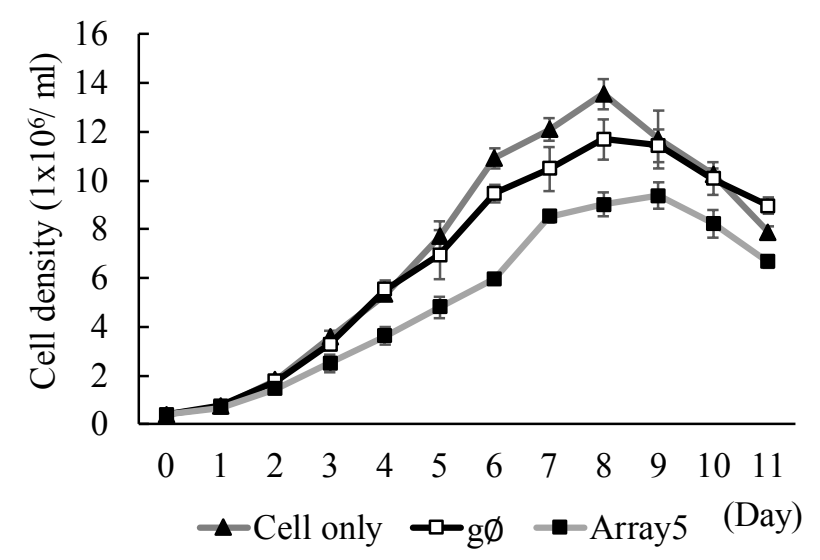

(E)

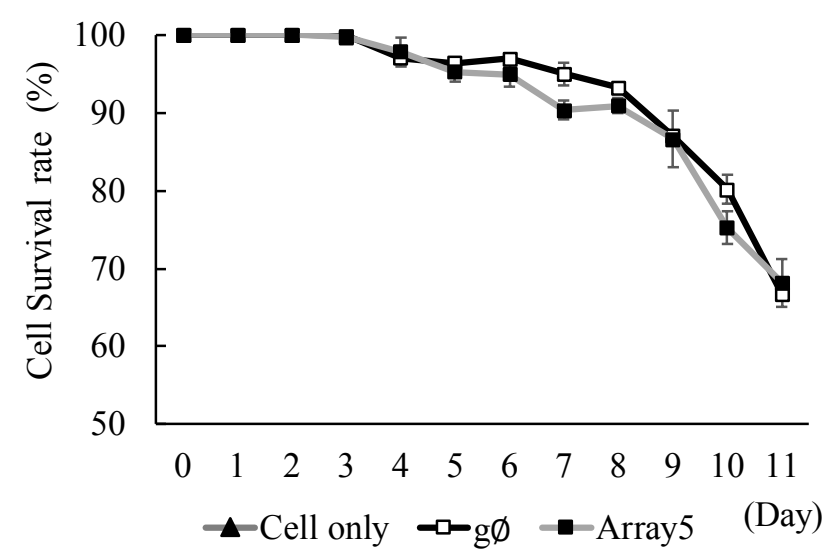

Figure S4. CRISPR-Cas13d can inhibit the expression of BAK, BAX and PDK1 in a multiplexing

manner in CHO $_{\text {IgG }}$. (A) Schematic illustration of pIS-Cas13dPZ-gRNA Array5. The vector was similar to pIS-Cas13dPZ-gRNA except that the gRNA was an array with one gRNA targeting BAK, two gRNA targeting BAX and two gRNA targeting PDK1. (B) $\mathrm{CHO}_{\mathrm{IgG}}$ was transfected with pIS-Cas13dPZ-gRNA Array5 (Array5 group) or pIS-Cas13dPZ-gRNA $\varnothing$ (gØ group) and selected using Zeocin. Compared with the $\mathrm{g} \varnothing$ group, the expression in the Array 5 group was reduced to $\approx 0.3 \%$ for $\mathrm{BAK}, \approx 0.3 \%$ for $\mathrm{BAX}$ and $\approx 9 \%$ for PDK1. The data are representative of 3 independent culture experiments and confirm that CRISPR-Cas13d can effectively inhibit the expression of BAK, BAX and PDK1 in a multiplexing manner (B) and improved the IgG production in $\mathrm{CHO}_{\mathrm{IgG}}(\mathrm{C})$. (D) Cell density. (E) Cell viability. The data showed that simultaneous inhibition of BAK, BAX and PDK1 slightly slowed the cell growth (D), but did not compromise the cell viability (E). Importantly, the CRISPR-Cas13d engineering increased the IgG titer (C). 